\title{
Hybrid Genetic Algorithm With Haar Wavelet for Maximum Target Coverage Node Deployment in Wireless Sensor Networks
}

T. Ganesan, Koneru Lakshmaiah Education Foundation, Guntur, India

iD https://orcid.org/0000-0003-1926-0948

Pothuraju Rajarajeswari, Koneru Lakshmaiah Education Foundation, Guntur, India

\begin{abstract}
Wireless sensor networks (WSNs) are used in industrial applications and focused on target coverage and node connectivity based WSNs. The set of sensors and targets is placed in optimal position the target coverage and node connectivity achieving maximum with limited senor nodes. To resolve this problem, the proposed hybrid genetic algorithm combined with lifting wavelet multi-resolution principles for recognizing optimal position for sensors to cover entire targets present in the fields. The hybrid genetic algorithm randomly identifies each sensor position and 2D Haar lifting wavelet transform to improve the quality of target coverage by adjusting node position. The 2D Haar lifting decomposes the population matrix into the optimal position of sensors. Experimental results show the performance of the proposed hybrid genetic algorithm and fast local search method compared with available algorithms improves the target coverage and the number of nodes with varying and fixed sensing ranges with a different region.
\end{abstract}

\section{KEYWORDS}

Genetic Algorithm, Haar Lifting Wavelet Transform, Node Connectivity, Optimal Sensor Placement, Target Coverage, Wireless Sensor Networks

\section{INTRODUCTION}

The advent which enabled their deployment in wireless sensor networks (WSNs) consists of mobile and stationary sensor nodes that are spatially sensing and processing the data. It is used for monitoring physical and interacting with the surrounding environment using multiple tiny sensors (Chamam and Pierre, 2009), (Yoon and Kim, 2013), (Ouchitachen et al., 2016). Sensor nodes are operating on battery power, sensors used in the unreachable area for a long period are not chargeable and unrepairable (Rajesh and Ranjitha, 2019), (Bhatia et al., 2016). In the last few years, many kinds of research have established to extend the node lifetime (Ouchitachen et al., 2016). Sensors act differently depending on situations while performing the intended application (Carrabs et al., 2015).

This article, published as an Open Access article on April 23, 2021 in the gold Open Access journal, Journal of Cases on Information Technology (JCIT) (converted to gold Open Access January 1, 2021), is distributed under the terms of the Creative Commons Attribution License (http://creativecommons.org/licenses/by/4.0/) which permits unrestricted use, distribution, and production in any medium, provided the author of the original work and original publication source are properly credited. 
Sensors mainly depend on the environmental application that differs from network deployment and the rate of energy depletion (Rajesh and Vinaygasundaram, 2014) (Chamam and Pierre, 2009), (Ghosal and Halder, 2015). There are two types of node deployment in WSNs, i.e., random, and deterministic node deployment (Ghosal and Halder, 2015). But the deployment of the hybrid networks is combined with mobile static sensors. In general, during configuration static sensor networks deployment is considered static or dynamic (Abdelkhalek et al., 2015), (Vilela et al., 2016). Random deployment mostly used in inaccessible environmental regions like the deep sea, volcanoes, very dangerous forest area, etc., where nodes are dropped by helicopter (Ghosal and Halder, 2015).

While deploying deterministic sensors focus on well planned accessible regions to meet predefined objectives like energy conservation, the number of nodes, the position of nodes, application of nodes, and network lifetime (Yoon and Kim, 2013), (Zhang et al., 2016). The genetic algorithm (GA) is also used for optimal sensor placement for static and dynamic networks. The main objectives for optimal sensor deployment enhance or limit the mobility or energy for increasing network lifetime, sensing range to cover or monitor the maximum number of targets or objects, communication range to interact with nodes (Vilela et al., 2016). The major aspects to be resolved while deploying node in an optimal position can be achieved by a limited number of sensors to cover the maximum number of targets with node connectivity.

The main contribution of this paper is as follows, initially, sensor nodes are deployed randomly in the monitoring fields to measure the quality of the number of target coverage or monitor. Based on the analysis the hybrid GA is to identify the optimal position of the sensor for modifying crossover and mutation points. A hybrid framework that consists of a GA based heuristic searches evolutionary algorithm which compromises many complex optimization problems successfully. The implementation of proposed hybrid GA is presented step by step, including initial population matrix, evaluation of fitness function for that matrix, the genetic operation like crossover and mutation, and the scheduling transition for lifting. Then local enhancement is done by a multiresolution analysis applying a lifting based wavelet transform for adjusting the GA population matrix.

The rest of this paper structured as follows. In Section 2, discuss the literature review provided for optimal sensor deployment coverage consent. Section 3 follows problem formation based on a network model and quality measure by the number of target coverage and number of node connectivity. Section 4 is about multi-resolution analysis in the form of a lifting scheme-based matrix decomposition. Section 5 deals with the proposed hybrid genetic algorithm-based node deployment in a population matrix format and enhanced by two dimensions Haar lifting wavelet transform (2DHLWT). Section 6 is describing the simulation results under ideal, more realistic scenarios and the quality of the number of target coverage or monitor. Finally, this paper concludes with the scope of the work.

\section{LITERATURE REVIEW}

Recently many researchers have developed different techniques to improve the quality of target coverage, node connectivity, and lifetime maximization. One of the most significant factors will directly affect coverage, node connectivity, and network lifetime by node placement.

The authors (Alia and Al-Ajouri, 2017) proposed maximum network coverage and minimum network cost are to be achieved by Harmony algorithm where it identifies the total number of sensors required and its position. The author divides the coverage probability into fully covered, partially covered, and uncovered. The quality of the target cover is measured by Harmony search parameters. The authors (Hu et al., 2010) applied forward encoding scheme-based Hybrid Genetic Algorithm (HGA) and scheduling transition for point coverage and area coverage (Katti, 2019), (Hanh et al., 2019) in disjoint set covers. The different features of HGA applied in the chromosomes of the population for effective scheduling transition. The novel forward encoding maximizes the gene value in all chromosomes for the quality of target coverage. The primary task of scheduling sensors transitions is to change unique incomplete set covers to complete set covers. 
The randomly deployed sensors are moving inside the field and to adjust its sensing range. The authors (Abo-Zahhad et al., 2016) proposed a model for identifying the optimal position for mobile sensors by redeploying and aims to network coverage and less energy dissipation for mobility and sense of sensors. The author presents centralized immune Voronoi deployment having two different phases, the initial antibody population having all the sensor location to be evaluated and sensing range are calculated for all the sensors, to identify and select the best antibodies to be replicated. The same thing is to be done in phase 2 with binary antibodies to find optimal nodes to cover the fields.

The author considered the k-coverage problematic for many sensor placements schemes. The authors (Ashouri et al., 2012) explored the number of disjoint set k-coverage to the extent of the lifespan. The target is covered by the very least number of sensors called as a critical target (Katti, 2019). The same as the set of sensors covering the least covered target is called critical sensors. The author defined the triple parameters $\mathrm{S}, \mathrm{T}, \mathrm{C}$ where $\mathrm{S}, \mathrm{T}$ as sensor set and target set, $\mathrm{C}$ is a coverage relation to use any $\mathrm{k}$ values for the maximum number of disjoint covers. The authors (Gupta et al., 2015) used GA based node placement, it delivers k-coverage of the set of target covers and m-connectivity for nodes. The coverage and connectivity problem to be stated as three Boolean variables called target cover, node-connect, and potential position.

The authors (Liao and Ting, 2018) proposed a novel memetic algorithm based on an integer coded genetic algorithm with some local search operation for set k-cover problem. While placing the sensor the tight upper bound value is derived to integer represent. Here crossover and mutation operation are used to separate the critical sensors. The recycling operator discovers the redundant sensors, noncritical sensors in all groups, and randomly dispatch this into groups to form the covers.

The evolutionary algorithm solves many mathematical optimization problems (Hanh et al., 2019). The authors (Chen et al., 2015) organize the coverage methods for disjoints set algorithms, nondisjoints set algorithms, and hybrid memetic frameworks algorithms. The authors proposed disjoint set covers (DSC) and dynamic coverage maintenance (DCM) for network lifetime maximization. The hybrid memetic algorithms obtain the coverage results while finding the maximum number of DSC. The heuristic recursive algorithm (HRA) is used to run and activate a specific number of nodes to patch coverage holes that may cause the node failure and energy depletion of nodes. The authors (Vijayaraju et al., 2017) proposed a hybrid memetic algorithm to generate a random population matrix containing sensor positions. This population matrix is decomposing for multi-resolution analysis to find optimal values. Here the 2D discrete Haar wavelets transform are applied for local enhancement to adjust the sensor position into optimal one.

The genetic algorithm based on a random population generation containing a sensor position (Ganesan and Rajarajeswari, 2019a). This sensor position is to be undergone to multilevel decompose using lazy lifting (Ganesan and Rajarajeswari, 2019b) and CDF 5/3 lifting (Ganesan et al., 2021) based wavelet transforms to adjust the sensor position to cover maximum targets. While using the lifting scheme the local search operation to be fastened and to find the optimal sensor position.

\section{PROBLEM FORMATION}

\subsection{Network Model}

In this division, the network model formulates the coverage-connectivity-energy problem as a mathematical problem. Define the sensorfield size is Ax A contains N sensors $S=\left\{S_{1}, S_{2}, S_{3}, \ldots S_{i}, \ldots S_{N}\right\}$ with the sensing range radius set $R_{S}=\left\{R_{S 1}, R_{S 2}, R_{S 3}, \ldots R_{S i}, \ldots . R_{S N}\right\}$ whereas $R_{S i} \in\left(R_{S_{\min }}, R_{S_{\max }}\right)$. All the sensors sensing range is to be adjusted within the $\left(R_{S_{\min }}, R_{S_{\max }}\right)$. The communication range radius of each sensor set $R_{C}=\left\{R_{C 1}, R_{C 2}, R_{C 3}, \ldots R_{C i}, \ldots . R_{C N}\right\}$ which guarantees the network connectivity of nodes at least by $R_{C i} \geq 2 R_{S i}$. Given $\mathrm{N}$ sensors randomly distributed to monitor the M targets $T=\left\{T_{1}, T_{2}, T_{3}, \ldots . T_{i}, \ldots . T_{M}\right\}$ which are available in the same region, $\mathrm{A} \times \mathrm{A}$. Target $\mathrm{T}_{\mathrm{i}}$ is 
said to be covered or monitored if it is available in the sensing range $\mathrm{R}_{\mathrm{Si}}$ of at least one sensor. All the sensors are partition into several groups for the target covers. Where cover is defined as a sensor group that can cover all the targets. When the available sensors count is more than the available targets, every sensor may be covered to all targets. But, here $N<M$ is a lesser amount of some sensors to cover the maximum number of targets. It leads to redundant covers of targets and some sensors cover non-redundant targets.

\subsection{Coverage and Connectivity Rate Calculation}

This subsection provides the sensor $\mathrm{S}_{\mathrm{i}}$ and $\mathrm{S}_{\mathrm{j}}$ is randomly placed in the region $\left(x_{i}, y_{i}\right)$ and $\left(x_{j}, y_{j}\right)$ with sensing range, $\mathrm{R}_{\mathrm{Si}}$ and $\mathrm{R}_{\mathrm{Sj}}$ respectively to cover the target $\mathrm{T}_{\mathrm{t}}$ which is available in the region $\left(x_{t}, y_{t}\right)$. The sensor $\mathrm{S}_{\mathrm{i}}$ monitors the target $\mathrm{T}_{\mathrm{t}}$ and $\mathrm{S}_{\mathrm{i}}$ connected to $\mathrm{S}_{\mathrm{j}}$ when it satisfies the following Euclidean distance-based condition (1) and (2):

$$
\operatorname{Cov}\left(S_{i}, T_{t}\right)=\left\{\begin{array}{l}
1, \operatorname{dist}\left(S_{i}, T_{t}\right) \leq R_{S i}, \forall i, 1 \leq i \leq N, \forall t, 1 \leq t \leq M \\
0, \text { otherwise }
\end{array}\right.
$$

$\operatorname{Con}\left(S_{i}, S_{j}\right)=\left\{\begin{array}{l}1, \operatorname{dist}\left(S_{i}, S_{j}\right) \leq R_{C i}, \forall i, 1 \leq i \leq N, \forall j, 1 \leq j \leq N \text { and } i \neq j \\ 0, \text { otherwise }\end{array}\right.$

The potential position of each sensor $\mathrm{P}_{\mathrm{Si}}$ is identified by coverage of targets and connectivity of sensors when the following constraint is satisfied (3) it can be represented in the form of the given matrix:

$$
\begin{aligned}
& P_{S i}=\left\{\begin{array}{l}
1, \operatorname{Cov}\left(S_{i}, T_{t}\right)=1 \text { and } \operatorname{Con}\left(S_{i}, S_{j}\right)=1 \\
0, \text { otherwise }
\end{array}\right. \\
& P_{S_{N} x T_{M}}=\left[\begin{array}{llll}
P_{1,1} & P_{1,2} & \ldots . & P_{1, M} \\
P_{2,1} & P_{2,2} & \ldots . . & P_{2, M} \\
\ldots . . & \ldots . . & \ldots . . & \ldots . . \\
P_{N, 1} & P_{N, 2} & \ldots . . & P_{N, M}
\end{array}\right]
\end{aligned}
$$

The matrix can be represented as total target coverage by doing a column sum of the matrix while deploying all sensors are defined as Qoc (\%) is referred to as the number of target object covered by using equation (4). Whereas to define the lifetime of every sensor node is $B S_{i}^{l}=B S_{i} / E S_{i}$. Where $\mathrm{BS}_{\mathrm{i}}$ is the initial battery power of sensor node $\mathrm{S}_{\mathrm{i}}$, and $\mathrm{ES}_{\mathrm{i}}$ is the rate of energy consumption of sensor $\mathrm{S}_{\mathrm{i}}$ (Mini et al., 2014). The network lifetime is achieved maximum by applying the upper bound configuration:

$$
U=\min \left[\frac{\sum_{i=1}^{N} P_{S i} x B S_{i}^{l}}{M}\right]
$$


$\operatorname{Qoc}(\%)=\frac{\sum_{j=1}^{M}\left[\frac{\sum_{i=1}^{N} P_{i, j}}{N}\right]}{M} x 100$

The illustration network of size $100 \times 100$ containing $M=16$ available targets is used in Figure 1 and $\mathrm{N}=8$ available number of sensors are randomly deployed in the same environmental region to monitor the targets is represented in Figure 2. The total coverage of all the sensors is acknowledged by equation(1). The coverage of all the sensors are $S 1=\{\}, S 2=\{\}, S 3=\{T 9\}, S 4=\{T 6, T 15\}$, $S 5=\{T 15, T 16\}, S 6=\{T 1\}, S 7=\{T 7, T 12\}$ and $S 8=\{T 3, T 8\}$. Randomly deployed targets and sensors shown in Figure 2 covers Qoc (\%) $56.25 \%$ of targets. But it does not assure the connectivity of the sensor, and the main objective is to cover total targets, which need an optimal number of sensor placement to monitor total targets.

\section{HAAR LIFTING WAVELET TRANSFORM}

The picture-perfect reconstruction filter pairs of $(\mathrm{h}, \mathrm{g})$ having similar low and high pass filters after lifting scheme. Initiated from lazy wavelet lifting to multiresolution analysis with some properties (Daubechies and Sweldens, 1998). The filter pairs (h, g) are complementary when the poly-phase matrix $\mathrm{P}(\mathrm{z})(6)$ has determinant matrix value 1 . Here any pairs of complementary filters $(\mathrm{h}, \mathrm{g})$ are factoring into lifting steps. To execute the Euclidean algorithm, start from even and odd values of $\mathrm{h}_{\mathrm{e}}(\mathrm{z})$ and $\mathrm{h}_{\mathrm{o}}(\mathrm{z})$ (Hasan and Wahid, 2016), (Hu and Jong, 2013):

Figure 1. Randomly deployed targets

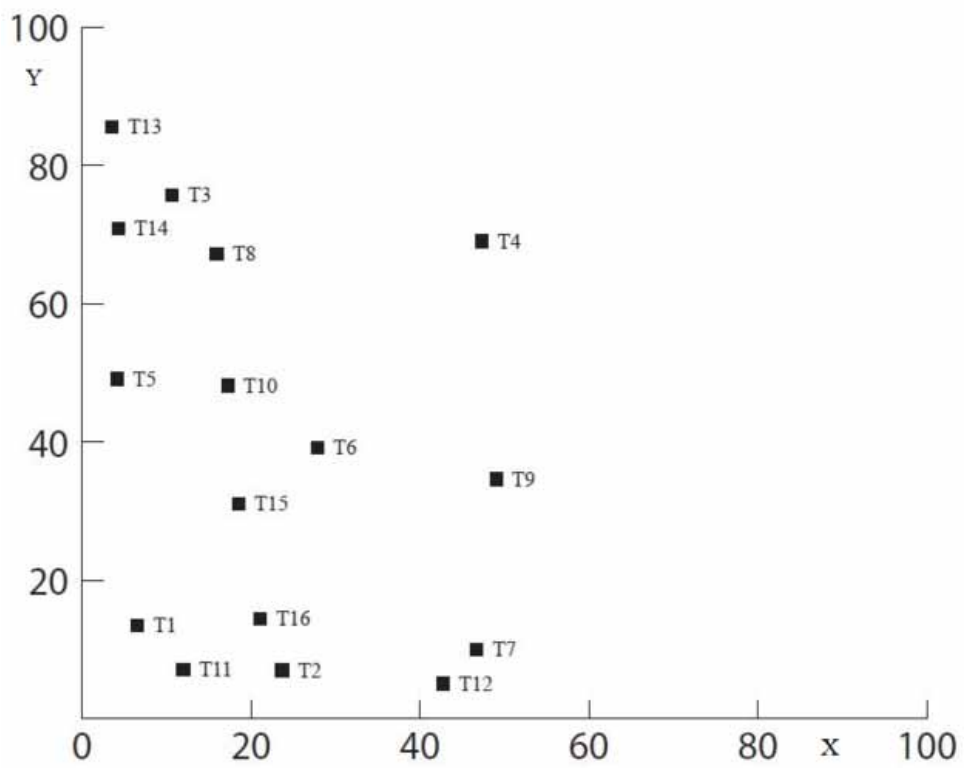




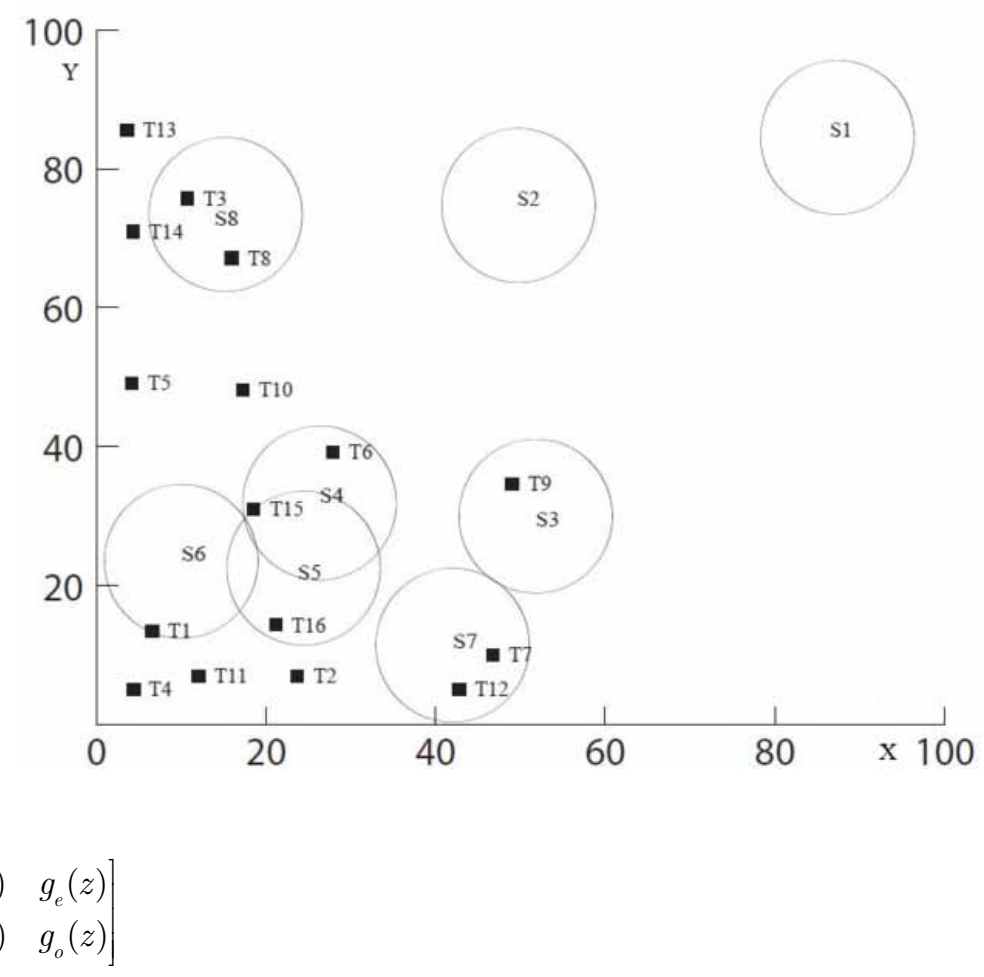

Un-Normalized Haar wavelet (Bamerni and Sulaifanie, 2019) is having $h(z)=1+z^{-1}$ synthesis low pass filter (SLPF), $\tilde{h}(z)=1 / 2+1 / 2 z^{-1}$ analysis low pass filter (ALPF), $\tilde{g}(z)=-1+z^{-1}$ analysis high pass filter (AHPF), and $g(z)=-1 / 2+1 / 2 z^{-1}$ synthesis high pass filter (SHPF), while executing the Euclidean algorithm the poly-phase matrix is given below:

$$
P(z)=\left[\begin{array}{cc}
1 & -1 / 2 \\
1 & 1 / 2
\end{array}\right]=\left[\begin{array}{cc}
1 & 0 \\
1 & 1
\end{array}\right]\left[\begin{array}{cc}
1 & -1 / 2 \\
0 & 1
\end{array}\right]
$$

In general lifting operations are done in three steps split, predict, and update. The initial operation split is done by separate even and odd data (Hasan and Wahid, 2016), (Hu and Jong, 2013). Predict and update operation; the data to get the detail and approximate data. Haar wavelet multiresolution analysis is done in the given equation (8) to (11) to produce the detail and approx. data for each level as shown in Figure 3. The input of $X(n)$ is split into an even $\left(X_{21}\right)$ and odd $\left(X_{21+1}\right)$ half sampling sequence. Here the odd component is predicted by even component through prediction operator, then the detailed output is $d_{l}^{1}$. The detailed input $d_{l}^{1}$ is updating through an update operator to get an approximate output $S_{l}^{1}$ :

$S_{l}^{0}=X_{2 l}$ 


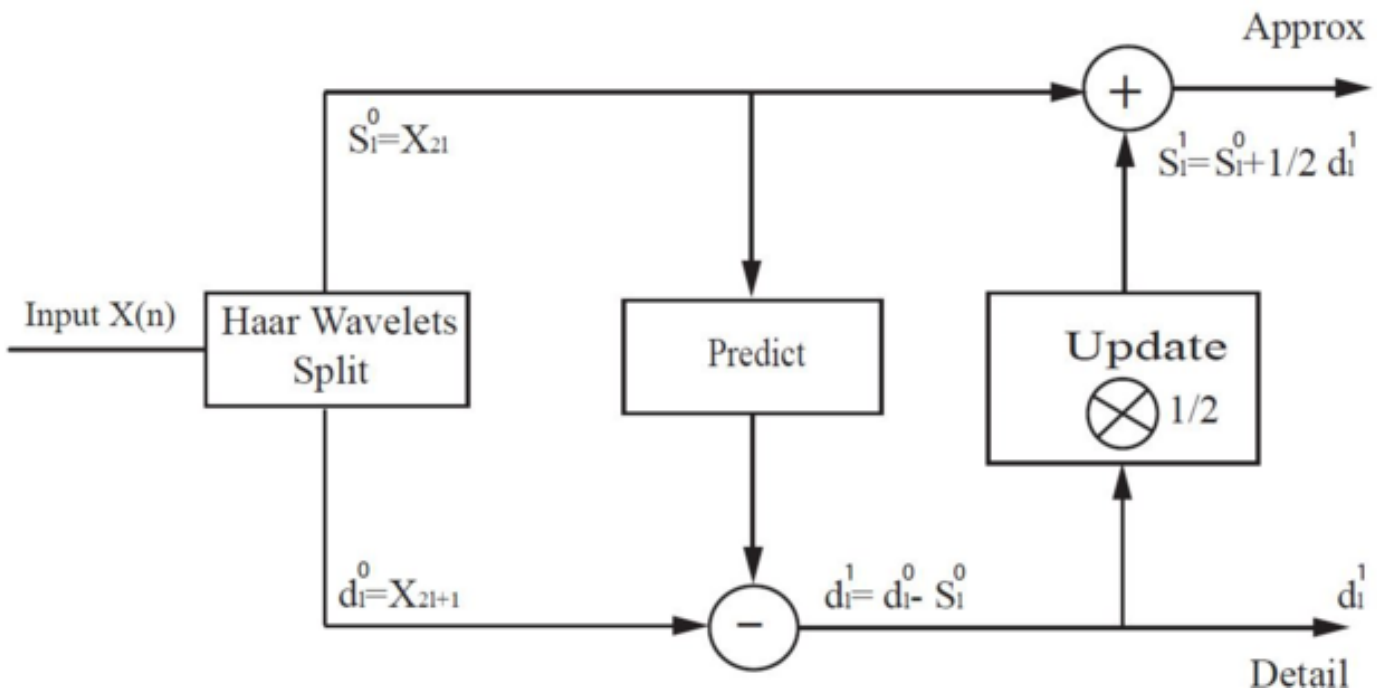

$d_{l}^{0}=X_{2 l+1}$

$d_{l}^{1}=d_{l}^{0}-S_{l}^{0}$

$S_{l}^{1}=S_{l}^{0}+1 / 2 d_{l}^{1}$

Here input data $\mathrm{N} \times \mathrm{N}$ two-dimensional matrix is applied first in row-wise followed by columnwise by Haar lifting wavelet transform represented in Figure 4. to produce LL approximation, LH, $\mathrm{HL}$, and $\mathrm{HH}$ detailed components.

The $\mathrm{N} \times \mathrm{N}$ size of the LL matrix is decomposed into level by level by applying conventional 2DHLWT produced N/2 $\times \mathrm{N} / 2 \mathrm{LL}$ for the next level. After level 1, again 2DHLWT is applied to get $\mathrm{N} / 4 \times \mathrm{N} / 4 \mathrm{LL}$ matrix for level 2. N/4 x N/4 matrix is decomposed to get N/8 x N/8 LL. This process will be repeated up to $\mathrm{N}=2^{\mathrm{j}}$ times and represented in Figure 5.

\section{GA-2D HAAR LIFTING WAVELET BASED OPTIMAL SENSOR PLACEMENT}

The general search problem solution to be optimized by one of the evolutionary algorithms is the Genetic algorithm (GA). Its initial population contains randomly generated values of sensor position called chromosome in the matrix rows.

Randomly generated values are 0 to A x A whereas $100 \times 100$. The initial population is evaluated by fitness function (5) by converting sensor position into coordinates:

$$
X=a b s\left(P_{i, j} / A\right) \text { and } Y=\left(P_{i, j}\right) \bmod (A)
$$




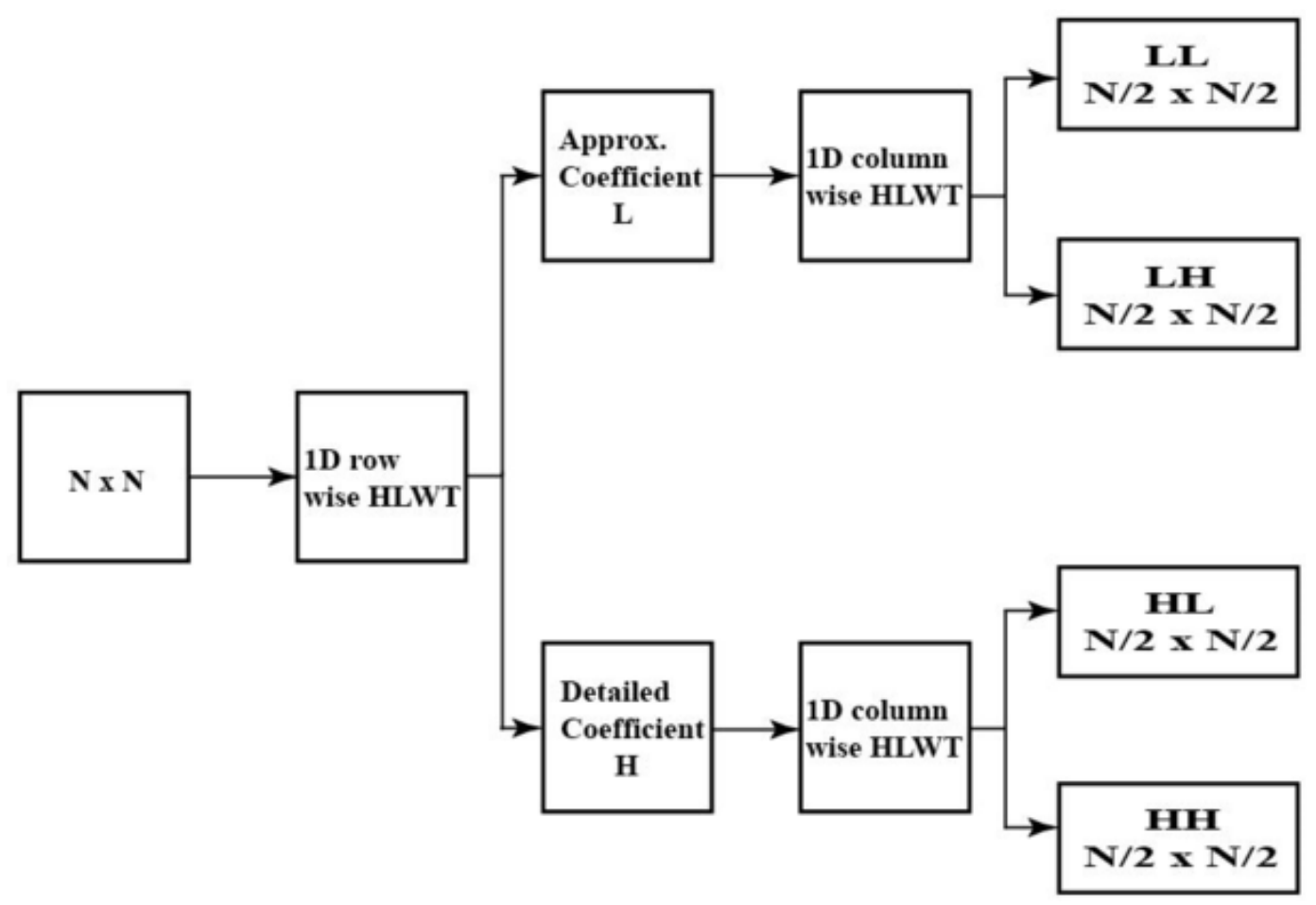

Once the initial population is evaluated by the fitness function, GA undergoes crossover, and mutation. Crossover is used to exchanges the genes among two chromosomes. Here having one or single-point crossover to be done for exchanging genes. GA generates a random value for a one-point crossover. After crossover, offspring are used to change some randomly generated gene values for mutation. Once the mutation is over the local search of the Haar lifting wavelet enhances the solution by adjusting the offspring values for the optimal position of sensors as represented in Figure 6 . The framework of proposed optimal sensor placement is in given Algorithm 1:

pop $1=\left[\begin{array}{llllllll}9081 & 4878 & 5236 & 2931 & 2330 & 1236 & 4213 & 1168 \\ 7235 & 3436 & 6543 & 7678 & 3233 & 5667 & 8789 & 2312 \\ 786 & 1012 & 1567 & 5532 & 4666 & 7889 & 9898 & 3234 \\ 7989 & 1223 & 3318 & 8582 & 8189 & 9163 & 7371 & 6823 \\ 9136 & 3267 & 4598 & 5832 & 983 & 2312 & 6543 & 9656 \\ 657 & 1777 & 1586 & 3156 & 7237 & 7447 & 2323 & 5646 \\ 578 & 3562 & 6587 & 5545 & 4555 & 6571 & 8381 & 8682 \\ 8321 & 7980 & 5062 & 4045 & 6380 & 2390 & 973 & 5470\end{array}\right]$


Figure 5. Haar Lifting Decompose matrix

\begin{tabular}{|c|c|c|c|c|c|c|c|c|c|c|c|c|c|c|c|}
\hline & & & put $=$ Y & $z(2 n)$ & & & & & & & evel 1 & $-\mathrm{Coh}$ & $\operatorname{umn} \nabla$ & Wise & \\
\hline LL & LL & LL & LL & LL & LL & LL & $L L$ & LL & HL & LL & $\mathrm{HL}$ & LL & HL & $L L$ & HL \\
\hline LL & LL & $L L$ & LL & LL & LL & LL & LL & LH & HH & LH & $\mathrm{HH}$ & $\mathbf{L H}$ & $\mathrm{HH}$ & $\mathrm{LH}$ & $\mathrm{HH}$ \\
\hline LL & LL & $\mathrm{LL}$ & LL & LL & LL & LL & LL & LL & HL & LL & $\mathrm{HL}$ & LL & HL & LL & HL \\
\hline LL & LL & $\mathbf{L L}$ & LL & LL & LL & LI & LL & LH & HH & LH & HH & LH & $\mathrm{HH}$ & LH & HH \\
\hline $\mathrm{LL}$ & LL & LL & LL & LL & LL & LL & LL & LL & HL & $\mathrm{LL}$ & HL & LL & $\mathrm{HL}$ & $L L$ & HL \\
\hline LL & LL & $L L$ & LL & LL & LL & LL & LL & LH & HH & $\mathrm{LH}$ & HH & $\mathbf{L H}$ & $\mathrm{HH}$ & $\mathrm{LH}$ & $\mathrm{HH}$ \\
\hline LL & LL & $\mathbf{L L}$ & LL & LL & LL & LL & LL & LL & HL & LL & HL & LL & HL & $L L$ & HL \\
\hline LI & LL & LI & LL & LL & LI & LL & LL & LH & HH & LH & $\mathrm{HH}$ & LH & HH & $\mathrm{LH}$ & $\mathrm{HH}$ \\
\hline & & & & & & & & & & & $y$ & 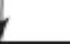 & & & \\
\hline & & & evel 3 & - Col & umn V & Nise & & & & & evel 2 & - Colu & $\mathrm{mnn} V$ & Vise & \\
\hline LL & HL & $\mathrm{HL}$ & HL & $\mathrm{HL}$ & HL & HL & HL & LL & $\mathrm{HL}$ & $\mathrm{HL}$ & $\mathrm{HL}$ & LL & HL & HL & HL \\
\hline $\mathrm{LH}$ & HH & LH & HH & $\mathrm{LH}$ & HH & LH & $\mathrm{HH}$ & LH & HH & LH & HH & LH & $\mathrm{HH}$ & $\mathrm{LH}$ & HH \\
\hline $\mathrm{LH}$ & HL & $\mathrm{HH}$ & $\mathrm{HL}$ & $\mathbf{L H}$ & HI & $\mathrm{HH}$ & $\mathrm{HL}$ & $\mathbf{L H}$ & HL & $\mathrm{HH}$ & HL & LH & HI & $\mathrm{HH}$ & HL \\
\hline LH & $\mathrm{HH}$ & LH & HH & $\mathbf{L H}$ & $\mathrm{HH}$ & LH & $\mathrm{HH}$ & LH & HH & $\mathbf{L H}$ & $\mathrm{HH}$ & $\mathbf{L H}$ & $\mathrm{HH}$ & $\mathbf{L H}$ & HH \\
\hline LH & HL & $\mathrm{HL}$ & $\mathrm{HL}$ & $\mathrm{HH}$ & HL & HL & HL & LL & HL & HL & $\mathrm{HL}$ & $\mathbf{L L}$ & HL & $\mathrm{HL}$ & HL \\
\hline LH & $\mathrm{HH}$ & LH & $\mathrm{HH}$ & LH & $\mathrm{HH}$ & LH & $\mathrm{HH}$ & LH & $\mathrm{HH}$ & LH & $\mathrm{HH}$ & $\mathbf{L H}$ & HH & $\mathbf{L H}$ & $\mathrm{HH}$ \\
\hline LH & HL & $\mathrm{HH}$ & HL & LH & HL & $\mathrm{HH}$ & $\mathrm{HL}$ & LH & HL & $\mathrm{HH}$ & $\mathrm{HL}$ & LH & $\mathrm{HL}$ & $\mathrm{HH}$ & HL \\
\hline LH & $\mathrm{HH}$ & $\mathbf{L H}$ & HH & LH & $\mathrm{HH}$ & LH & $\mathrm{HH}$ & $\mathrm{LH}$ & $\mathrm{HH}$ & $\mathrm{LH}$ & $\mathrm{HH}$ & $\mathbf{L H}$ & $\mathrm{HH}$ & $\mathbf{L H}$ & $\mathrm{HH}$ \\
\hline
\end{tabular}

pop $2=\left[\begin{array}{llllllll}8846 & 4590 & 2340 & 3888 & 4302 & 3781 & 7180 & 5240 \\ 850 & 4680 & 3057 & 9034 & 3409 & 1580 & 2003 & 1067 \\ 5003 & 5780 & 3098 & 679 & 4052 & 5040 & 2057 & 5305 \\ 5002 & 2012 & 1989 & 1990 & 4042 & 1991 & 2021 & 4590 \\ 9065 & 6640 & 6070 & 5460 & 3408 & 2379 & 4002 & 3045 \\ 7074 & 930 & 8074 & 7049 & 8905 & 1098 & 1005 & 4789 \\ 1877 & 5329 & 4280 & 5289 & 5034 & 1290 & 9076 & 3480 \\ 9667 & 4087 & 3287 & 676 & 5980 & 8092 & 7423 & 4490\end{array}\right]$

The initial population matrix is used to evaluate the fitness function of Qoc (\%) while converting into coordinates position: 
Figure 6. Flowchart for Process of GA+2DHLWT

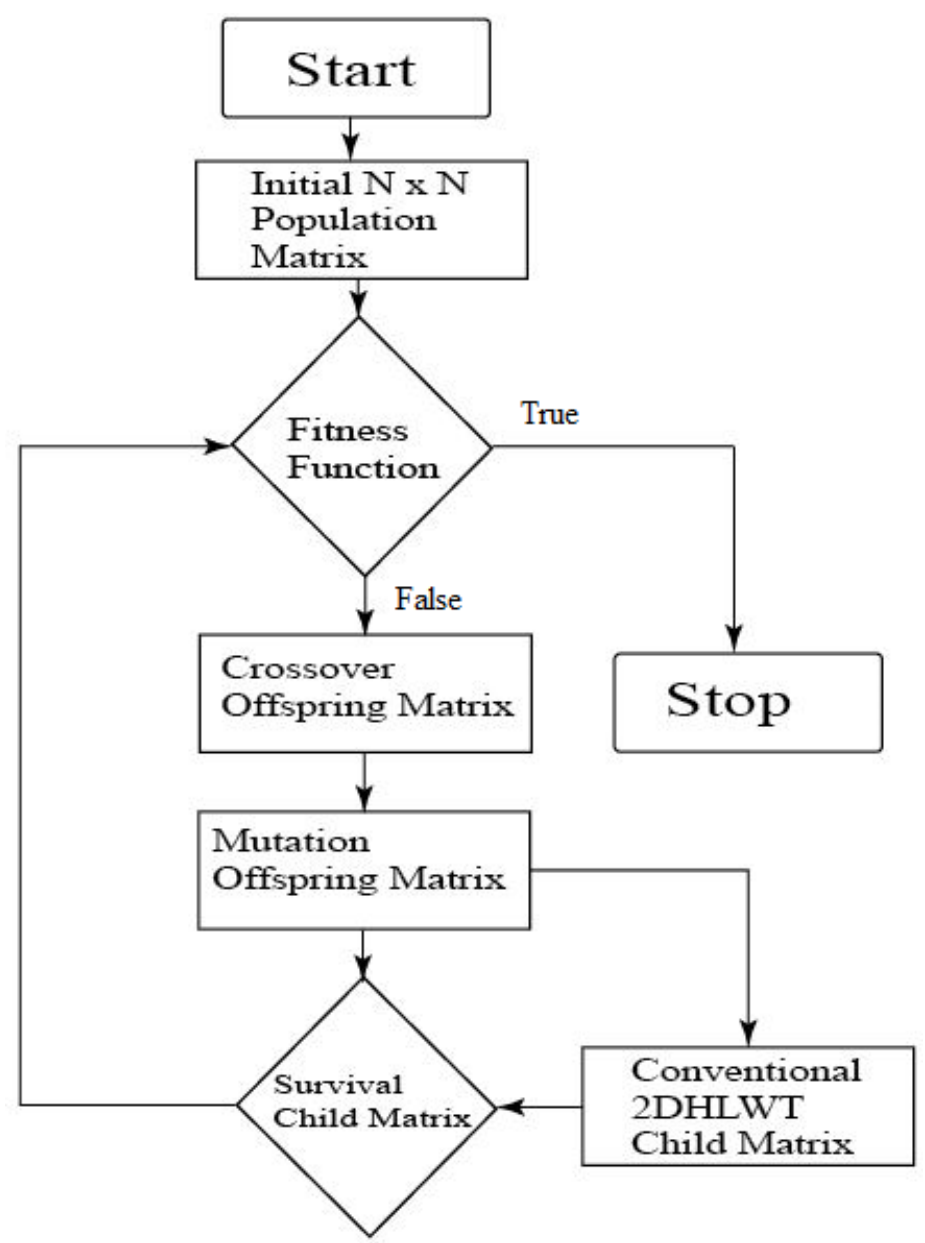

Algorithm 1. GA + Haar Lifting Wavelet transform for sensor placement

Input: $\mathrm{N}, \mathrm{M}, \mathrm{T}_{\mathrm{t}}, \mathrm{R}_{\mathrm{Si}}$ and $\mathrm{R}_{\mathrm{Ci}}$ entire sensor.

Output: optimal sensor position.

1: Start

2: $p o p=$ Randomly generated initial population matrix

3 : for $\mathrm{i}=1$ : gene:

while Fitness(pop)! =TRUE

offs_c=Crossover(pop).

offs_m=Mutation $($ offs_c $)$.

pop_ls=Local_Search_Haar_Lifting $\left(o f f s \_m\right)$.

pop=Survival (pop, pop_ls).

4: Display the position of the sensors in the matrix.

5: Stop 
pop $1=\left[\begin{array}{l}X \\ Y\end{array}\right]=\left[\begin{array}{llllllll}90 & 48 & 52 & 29 & 23 & 12 & 42 & 11 \\ 81 & 78 & 36 & 31 & 30 & 36 & 13 & 68\end{array}\right]$

pор $2=\left[\begin{array}{l}X \\ Y\end{array}\right]=\left[\begin{array}{llllllll}88 & 45 & 23 & 38 & 43 & 37 & 71 & 52 \\ 46 & 90 & 40 & 88 & 02 & 81 & 80 & 40\end{array}\right]$

The pop $1(\mathrm{X}, \mathrm{Y})$ coordinates are deployed into the region Figure 2. And fitness function is $56.25 \%$ while deploying the entire sensor only 9 targets are covered. Here randomly generated onepoint crossover is 5 and pop 1 and pop 2 will be changed:

offs $1=\left[\begin{array}{llllllll}9081 & 4878 & 5236 & 2931 & 2330 & 3781 & 7180 & 5240 \\ 7235 & 3436 & 6543 & 7678 & 3233 & 1580 & 2003 & 1067 \\ 786 & 1012 & 1567 & 5532 & 4666 & 5040 & 2057 & 5305 \\ 7989 & 1223 & 3318 & 8582 & 8189 & 1991 & 2021 & 4590 \\ 9136 & 3267 & 4598 & 5832 & 983 & 2379 & 4002 & 3045 \\ 657 & 1777 & 1586 & 3156 & 7237 & 1098 & 1005 & 4789 \\ 578 & 3562 & 6587 & 5545 & 4555 & 1290 & 9076 & 3480 \\ 8321 & 7980 & 5062 & 4045 & 6380 & 8092 & 7423 & 4490\end{array}\right]$

offs $2=\left[\begin{array}{llllllll}8846 & 4590 & 2340 & 3888 & 4302 & 1236 & 4213 & 1168 \\ 850 & 4680 & 3057 & 9034 & 3409 & 5667 & 8789 & 2312 \\ 5003 & 5780 & 3098 & 679 & 4052 & 7889 & 9898 & 3234 \\ 5002 & 2012 & 1989 & 1990 & 4042 & 9163 & 7371 & 6823 \\ 9065 & 6640 & 6070 & 5460 & 3408 & 2312 & 6543 & 9656 \\ 7074 & 930 & 8074 & 7049 & 8905 & 7447 & 2323 & 5646 \\ 1877 & 5329 & 4280 & 5289 & 5034 & 6571 & 8381 & 8682 \\ 9667 & 4087 & 3287 & 676 & 5980 & 2390 & 973 & 5470\end{array}\right]$

To obtain the optimal sensor position some of the genes in the chromosome are applied for mutation. Randomly generated mutation point is 7 : 


offs $1=\left[\begin{array}{llllllll}9081 & 4878 & 5236 & 2931 & 2330 & 3781 & 2441 & 5240 \\ 7235 & 3436 & 6543 & 7678 & 3233 & 1580 & 8083 & 1067 \\ 786 & 1012 & 1567 & 5532 & 4666 & 5040 & 3604 & 5305 \\ 7989 & 1223 & 3318 & 8582 & 8189 & 1991 & 5098 & 4590 \\ 9136 & 3267 & 4598 & 5832 & 983 & 2379 & 2442 & 3045 \\ 657 & 1777 & 1586 & 3156 & 7237 & 1098 & 7836 & 4789 \\ 578 & 3562 & 6587 & 5545 & 4555 & 1290 & 1006 & 3480 \\ 8321 & 7980 & 5062 & 4045 & 6380 & 8092 & 5890 & 4490\end{array}\right]$

offs $2=\left[\begin{array}{llllllll}8846 & 4590 & 2340 & 3888 & 4302 & 1236 & 7680 & 1168 \\ 850 & 4680 & 3057 & 9034 & 3409 & 5667 & 3230 & 2312 \\ 5003 & 5780 & 3098 & 679 & 4052 & 7889 & 3035 & 3234 \\ 5002 & 2012 & 1989 & 1990 & 4042 & 9163 & 6781 & 6823 \\ 9065 & 6640 & 6070 & 5460 & 3408 & 2312 & 8060 & 9656 \\ 7074 & 930 & 8074 & 7049 & 8905 & 7447 & 6089 & 5646 \\ 1877 & 5329 & 4280 & 5289 & 5034 & 6571 & 8902 & 8682 \\ 9667 & 4087 & 3287 & 676 & 5980 & 2390 & 5670 & 5470\end{array}\right]$

The local search of 2D Haar lifting wavelet transform (2DHLWT) decomposes the offspring 1 and offspring 2 level by level according to row-wise and column-wise and the final child 1 and child 2 are given in (20) and (21). After applying 2DHLWT, some of the values are identified as undesirable and out of the region. It shows that these values are more than $\mathrm{A} \times \mathrm{A}$, in this situation a simple component is applied to bring all the sensor positions into the region $P_{i, j}=a b s\left(P_{i, j}\right) \bmod (A x A)$ :

child $1=\left[\begin{array}{llllllll}4374 & 4001 & 718 & 585 & 546 & 101 & 577 & 2108 \\ 1644 & 404 & 3027 & 3440 & 649 & 3104 & 734 & 9815 \\ 2126 & 3270 & 2557 & 4614 & 1341 & 2912 & 1799 & 596 \\ 3707 & 6992 & 2400 & 1299 & 237 & 6572 & 389 & 2209 \\ 205 & 2374 & 141 & 1402 & 255 & 2371 & 120 & 1222 \\ 4984 & 6989 & 2844 & 336 & 2486 & 7535 & 3569 & 3650 \\ 1458 & 1321 & 115 & 1029 & 671 & 776 & 2966 & 537 \\ 6080 & 3325 & 1512 & 25 & 4313 & 4977 & 2947 & 3874\end{array}\right]$




child $2=\left[\begin{array}{llllllll}5030 & 213 & 1336 & 3762 & 829 & 404 & 687 & 3715 \\ 3953 & 8086 & 2931 & 4429 & 1769 & 5324 & 1653 & 5594 \\ 1466 & 1106 & 2348 & 1209 & 2001 & 4479 & 1262 & 120 \\ 1884 & 3767 & 101 & 2420 & 632 & 1284 & 3667 & 157 \\ 1506 & 4284 & 560 & 817 & 261 & 1277 & 2016 & 576 \\ 3850 & 3719 & 1796 & 415 & 5316 & 362 & 2990 & 2039 \\ 1983 & 1064 & 2593 & 801 & 353 & 1026 & 342 & 210 \\ 3274 & 9032 & 2803 & 3620 & 1617 & 5127 & 3222 & 20\end{array}\right]$

Again, every row of the child matrix is transformed into a sensor position as described in the above process and fitness function is evaluated in Figure 7. And Qoc (\%) of target coverage is achieved $81.25 \%$ of targets covered and connectivity is also achieved by 2DHLWT.

\section{SIMULATION RESULTS}

To evaluate the quality of the number of target coverage of the proposed method, a serious of simulations were conducted in the MATLAB R2012b, Intel i7 processor, $3.4 \mathrm{GHz}$ CPU, 8GB RAM, and Windows 7 environment to know the performance comparison. The simulation work is carried out with 4 to 256 sensors, and sensing ranges is varied from 125 to 225 , the communication range is defined as $2 \mathrm{R}_{\mathrm{Si}}$ based on sensor sensing range; total targets are limits to 10 to 450 and region to be $100 \times 100,500 \times 500$ and $1000 \times 1000$ to generate the random position of sensors. The number of initial populations is limited to 10 , one-point crossover and mutation are generated randomly in the simulation. The main objectives of the proposed method are to recognize the optimal sensor position to cover entire targets so that the performance measures Qoc (\%) of a different situation in fields.

Figure 7. GA+2DHLWT optimal Sensor placement

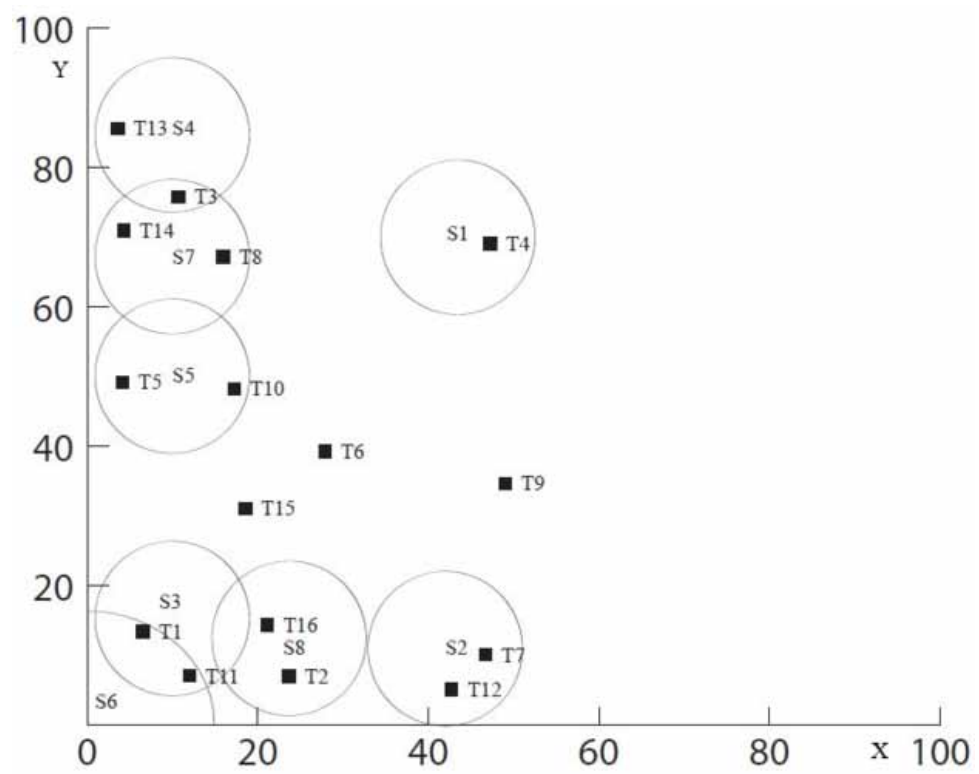


To analyze the performance of Qoc (\%) of the proposed method, the simulation to be performed for random deployment (RD), a genetic algorithm-based deployment (GAD), and GA with 2DHLWT (GHLD) deployment are used. Here the Qoc (\%) will depend only on fixed target positions, once the position is changing it is not possible to measure the quality of the number of targets covers. The performance of the proposed algorithm validates two different scenarios. One is the variation of targets and fixed sensing range of sensors; another is a fixed number of targets and variation of sensing ranges of sensors. The simulation environment is carried out by three different filed of A $\mathrm{x}$ A.

Qoc is identified for $100 \times 100,500 \times 500$ and $1000 \times 1000$ with different numbers of the sensor varies 4 to 256 and it depends on the number of targets, fixed sensing range $R_{\mathrm{Si}}=225$, and communication range of nodes are shown in Figure 8, 9 and 10. The average coverage of targets in $\mathrm{RD}$ is $60 \%$ to $70 \%$, GAD is $70 \%$ to $80 \%$ and GHLD is $80 \%$ to $90 \%$. Here RD and GAD placements cover a minimum number of targets while varying environment fields but the proposed algorithm GHLD covers a maximum number of targets in different fields.

The proposed method for fixed targets 400 and varying sensor ranges is 150,175 , and 200 validated in the field $1000 \times 1000$. The average Qoc of target coverage is RD in Figure 11. Is $40 \%$ to $50 \%$, GAD is $50 \%$ to $60 \%$ and GHLD is $70 \%$ to $85 \%$. Whereas, the proposed method covers the maximum number of targets while changing the sensing range and communication ranges of sensors.

\section{CONCLUSION}

WSNs are comprised of resource-constrained nodes while facing optimal sensor placement for target coverage. This paper represents a hybrid genetic algorithm approach with 2DHLWT for identifying the optimal sensor placement to cover up the maximum number of targets with a limited number of sensors. GA efficiently formulates sensor position and it can be adjusted through the local enhancement using 2DHLWT for identifying optimized sensor position to cover up entire targets. The proposed hybrid genetic algorithm with Haar lifting has been validated by a sequence of simulation outcomes with environment regions and different scenarios. The results attained from the hybrid algorithm overcomes the existing random and genetic algorithm-based deployment in terms of coverage by

Figure 8. Qoc generated for $100 \times 100, M=2 N$, and $R_{S i}=225$

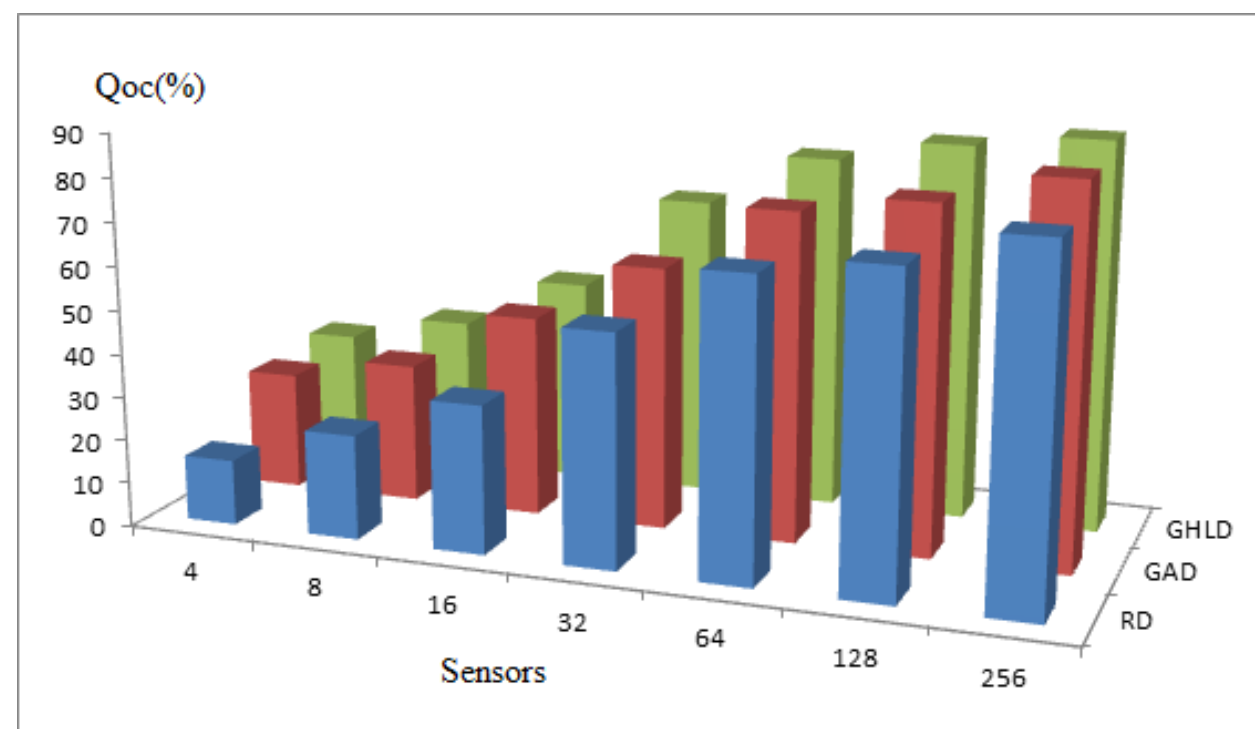


Figure 9. Qoc generated for $500 \times 500, M=2 N$, and $R_{S i}=225$

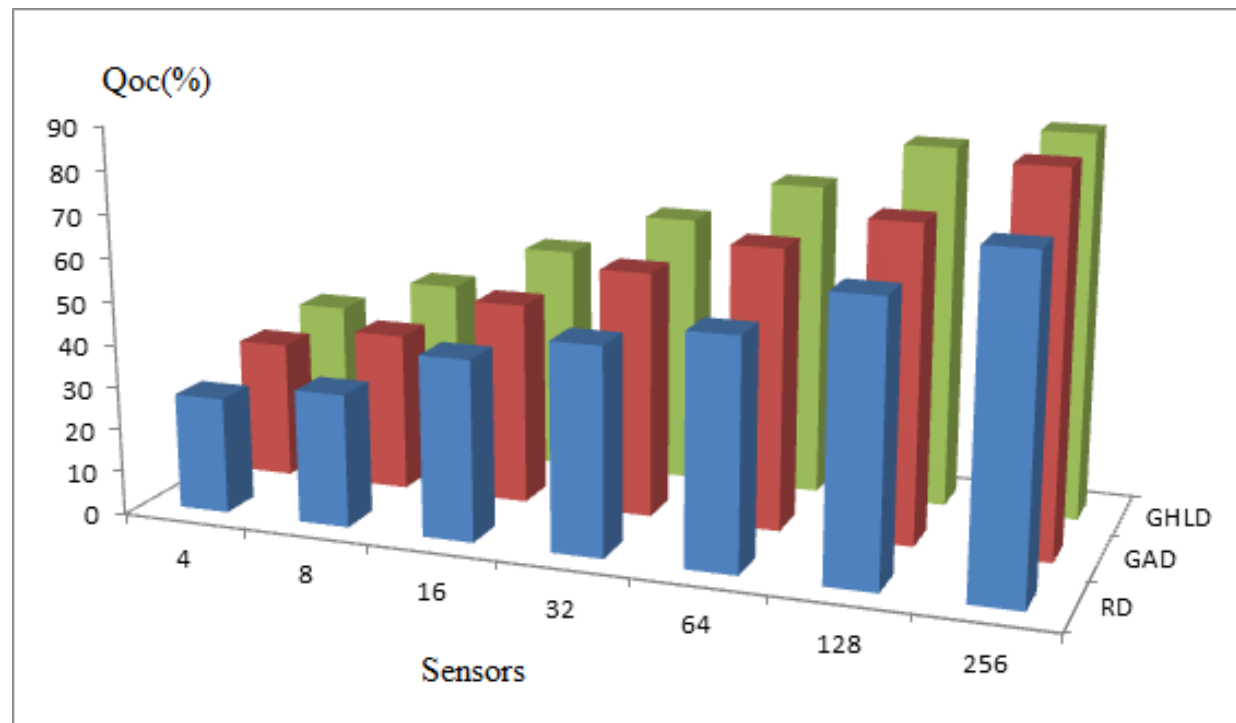

Figure 10. Qoc generated for $1000 \times 1000, M=2 N$, and $R_{\mathrm{Si}}=225$

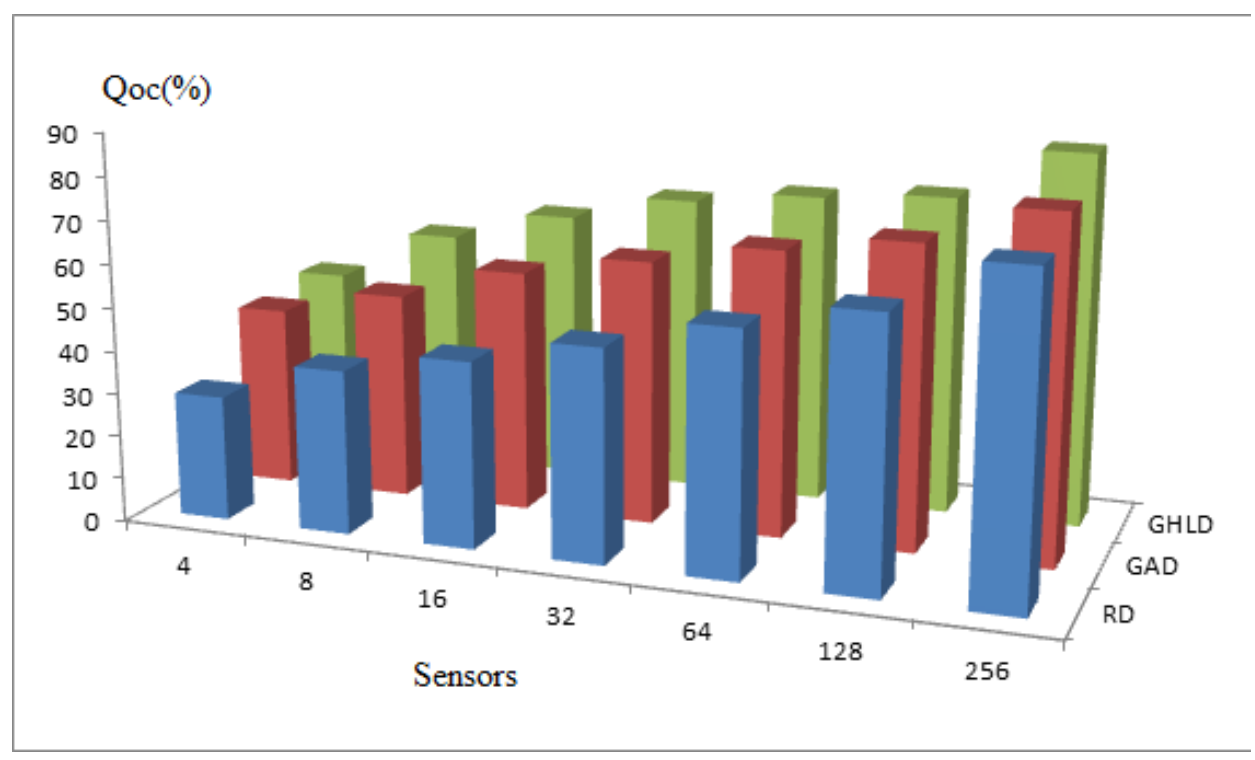

Figure 11. Qoc generated for $1000 \times 1000$, and $M=400$

\begin{tabular}{|c|c|c|c|c|c|c|c|c|c|}
\hline \multirow{3}{*}{ Sensors } & \multicolumn{9}{|c|}{ Qoc (\%) } \\
\cline { 2 - 10 } & \multicolumn{3}{|c|}{$\mathrm{R}_{\text {Si }}=150$} & \multicolumn{3}{c|}{ Rsis=175 } & \multicolumn{3}{c|}{$\mathrm{R}_{\text {sis }}=200$} \\
\cline { 2 - 10 } & RD & GAD & GHLD & RD & GAD & GHLD & RD & GAD & GHLD \\
\hline 4 & 22 & 27 & 34 & 27 & 32 & 42 & 29 & 34 & 47 \\
8 & 32 & 35 & 38 & 33 & 38 & 47 & 33 & 42 & 53 \\
16 & 34 & 37 & 43 & 38 & 46 & 52 & 42 & 47 & 58 \\
32 & 43 & 54 & 65 & 48 & 58 & 68 & 52 & 63 & 74 \\
64 & 53 & 68 & 76 & 57 & 72 & 79 & 60 & 75 & 85 \\
\hline
\end{tabular}


an optimal sensor position with limited sensors. In the future, aiming to study the different aspects of GA and Set K-cover problem with lifting wavelet-based sensor node deployment. The problem formation can be extended to dynamic nature with maximization of obstacle parameter in target coverage problems related to node connectivity of WSNs. 


\section{REFERENCES}

Abdelkhalek, O., Krichen, S., \& Guitouni, A. (2015). A genetic algorithm-based decision support system for the multi-objective node placement problem in next wireless generation network. Applied Soft Computing, 33, 278-291. doi:10.1016/j.asoc.2015.03.034

Abo-Zahhad, M., Sabor, N., Sasaki, S., \& Ahmed, S. M. (2016). A centralized immune-Voronoi deployment algorithm for coverage maximization and energy conservation in mobile wireless sensor networks. Information Fusion, 30, 36-51. doi:10.1016/j.inffus.2015.11.005

Ashouri, M., Zali, Z., Mousavi, S. R., \& Hashemi, M. R. (2012). New optimal solution to disjoint set k-coverage for lifetime extension in wireless sensor networks. IET Wirel. Sens.Syst., 2(1), 31-39. doi:10.1049/iet-wss.2011.0085

Bamerni, S. A., \& Kh, A. (2019). An efficient non-separable architecture for Haar Wavelet transform with lifting structure. Journal of Microprocessors and Microsystems, 71(1), 1-7. doi:10.1016/j.micpro.2019.102881

Bhatia, T., Kansal, S., Goel, S., \& Verma, A. K. (2016). A genetic algorithm-based distance-aware routing protocol for wireless sensor networks. Computers \& Electrical Engineering, 56, 1-15. doi:10.1016/j. compeleceng.2016.09.016

Carrabs, , \& Cerulli, , Ambrosio, \& Gentili. (2015). Maximizing lifetime in wireless sensor networks with multiple sensor families. Computers \& Operations Research, 60, 121-137. doi:10.1016/j.cor.2015.02.013

Chamam \& Pierre. (2009). On the Planning of Wireless Sensor Networks: Energy-Efficient Clustering under the Joint Routing and Coverage Constraint. IEEE Trans. on Mobile Computing., 8(8), 1077-1085.

Chen, C.-P., Mukhopadhyay, S. C., Chuang, C.-L., Lin, T.-S., Liao, M.-S., Wang, Y.-C., \& Jiang, J.-A. (2015). A Hybrid Memetic Framework for Coverage Optimization in Wireless Sensor Networks. IEEE Transactions on Cybernetics, 45(10), 2309-2321. doi:10.1109/TCYB.2014.2371139 PMID:25532143

Daubechies, I., \& Sweldens, W. (1998). Factoring Wavelet Transform into Lifting Steps. The Journal of Fourier Analysis and Applications, 4(3), 247-269. doi:10.1007/BF02476026

Ganesan \& Rajarajeswari. (2019a). Genetic Algorithm Based Optimization to Improve the Cluster Lifetime by Optimal Sensor Placement in WSN's. International Journal of Innovative Technology and Exploring Engineering, 8(8), 3400-3408.

Ganesan \& Rajarajeswari. (2019b). Genetic algorithm approach improved by 2D lifting scheme for sensor node placement in optimal position. Second International Conference on Intelligent Sustainable Systems, 1, $104-109$.

Ganesan, Rajarajeswari, Nayak, \& Bhatia. (2021). A novel genetic algorithm with CDF5/3 filter-based lifting scheme for optimal sensor placement. Int. J. Innovative Computing and Applications, 12(4), 1-10.

Ghosal, A., \& Halder, S. (2015). Lifetime Optimizing Clustering Structure Using Archimedes' Spiral-Based Deployment in WSNs. IEEE Systems Journal, 1-10.

Gupta, S. K., Kuila, P., \& Jana, P. K. (2015). Genetic algorithm approach for $k$-coverage and $m$ connected node placement in target-based wireless sensor networks. Computers \& Electrical Engineering, 1-13.

Hanh, N. T., Huynh, T. T. B., Hoai, N. X., \& Palaniswami, M. S. (2019). An efficient genetic algorithm for maximizing area coverage in wireless sensor networks. Journal of Information Science, 488(2), 59-75.

Hu, X.-M., Zhang, J., Yu, Y., Chung, H. S.-H., Li, Y.-L., Shi, Y.-H., \& Luo, X.-N. (2010). Hybrid Genetic Algorithm using a Forward Encoding Scheme for Lifetime Maximization of Wireless Sensor Networks. IEEE Transactions on Evolutionary Computation, 14(5), 766-780. doi:10.1109/TEVC.2010.2040182

Hu, Y., \& Jong, C. C. (2013). A Memory-Efficient High-Throughput Architecture for Lifting-Based MultiLevel 2-D DWT. IEEE Transactions on Signal Processing, 61(20), 4975-4987. doi:10.1109/TSP.2013.2274640

Katti. (2019). Target coverage in random wireless sensor networks using cover set. Journal of King Saud University-Computer and Information Sciences, 5(6), 1-13.

Liao, C.-C., \& Ting, C.-K. (2018). A Novel Integer-Coded Memetic Algorithm for the Set k-Cover Problem in Wireless Sensor Networks. IEEE Transactions on Cybernetics, 48(8), 2245-2257. doi:10.1109/ TCYB.2017.2731598 PMID:28829325 
Mehedi Hasan \& Wahid. (2016). Low-cost Architecture of Modified Daubechies Lifting Wavelets using Integer Polynomial Mapping. IEEE Transactions on Circuits and Systems II, 2(10), 1-5.

Mini, Udgata, \& Sabat. (2014). Sensor Deployment and Scheduling for Target Coverage Problem in Wireless Sensor Networks. IEEE Sensors Journal, 14(3), 636-644.

Moh'd Alia, O., \& Al-Ajouri, A. (2017). Maximizing Wireless Sensor Network Coverage with Minimum Cost Using Harmony Search Algorithm. IEEE Sensors Journal, 17(3), 882-896. doi:10.1109/JSEN.2016.2633409

Ouchitachen, H., Hair, A., \& Idrissi, N. (2016). Improved multi-objective weighted clustering algorithm in Wireless Sensor Network. Egyptian Informatics Journal.

Rajesh, \& Vinaygasundaram. (2014). Energy-Efficient Sub-Clustering with Optimized Processor Speed (Esops) in Wireless Sensor Networks. Journal of Theoretical and Applied Information Technology, 62(3), 812-818.

Rajesh, G., \& Ranjitha, R. (2019). A Cooperative Game Theoretic Approach for Congestion Management in 6LoWPAN. Journal of Information Science and Engineering, 35, 737-748.

Vijayaraju, P., Sripathy, B., Arivudainambi, D., \& Balaji, S. (2017). Hybrid Memetic Algorithm with TwoDimensional Discrete Haar Wavelet Transform for Optimal Sensor Placement. IEEE Sensors Journal, VOL., 17(7), 2267-2278. doi:10.1109/JSEN.2017.2662951

Vilela, J., Kashino, Z., Ly, R., Nejat, G., \& Benhabib, B. (2016). A Dynamic Approach to Sensor Network Deployment for Mobile-Target Detection in Unstructured, Expanding Search Areas. IEEE Sensors Journal, 16(11), 4405-4417. doi:10.1109/JSEN.2016.2537331

Yoon, Y., \& Kim, Y.-H. (2013). An Efficient Genetic Algorithm for Maximum Coverage Deployment in Wireless Sensor Networks. IEEE Transactions on Cybernetics, 43(5), 1473-1483. doi:10.1109/TCYB.2013.2250955 PMID:23757541

Zhang, Y.-H., Gongb, Y.-J., Gu, T.-L., Li, Y., \& Zhang, J. (2016). Flexible genetic algorithm: A simple and generic approach to node placement problems. Applied Soft Computing, 1-14.

T. Ganesan is currently working as an Assistant Professor in the Department of CSE at K L University (KLEF), India, where he is currently pursuing his PhD. He has completed his BTech and MTech from the Anna University, India. He published several research papers in the reputed journals particularly in the area of wireless sensor network. His research interests include wireless sensor network, genetic algorithm and machine learning.

Pothuraju Rajarajeswari received her Doctorate in Computer Science and Engineering and PhD in CSE in 2012 from the Acharya Nagarjuna University. Currently she is working as a Professor in the Department of CSE in KL University (KLEF). She has professional memberships in FIETE, MISTE, MIACSIT in International Association of Computer Science and Information Technology, and MIAENG in International Association of Engineers. She has 19 years of experience in teaching computer subjects for BTech, MCA and MTech Postgraduate students. Her research interests are bioinformatics, data mining, artificial intelligence, and data science. 